\title{
A good asthma catch by professional fishing
}

\author{
Peter J. Sterk \\ Affiliation: Dept of Respiratory Medicine, Academic Medical Centre, University of Amsterdam, Amsterdam, \\ The Netherlands. \\ Correspondence: Peter J. Sterk, Dept of Respiratory Medicine, F5-258, Academic Medical Centre, University \\ of Amsterdam, PO Box 22700, NL-1100 DE Amsterdam, The Netherlands. E-mail: p.j.sterkdaamc.nl
}

@ERSpublications

Broad metabolomic profiling of patients with asthma allows discovery of previously unidentified disease mechanisms http://ow.ly/dHpo309Kf0G

Cite this article as: Sterk PJ. A good asthma catch by professional fishing. Eur Respir J 2017; 49: 1602564 [https://doi.org/10.1183/13993003.02564-2016].

How long have we all been circling around asthma in order to capture its driving mechanisms? It is quite confronting that, despite excellent research during the past half century, we are still largely ignorant with regard to the biological pathways that cause and maintain this highly prevalent disease. Fortunately, asthma patients around the world are experiencing, daily, the benefits of solid research, which has led to very effective treatment options, at least for those that have access to good clinical care. Hence - yes - we have learned how to suppress the disease in most (but not all) patients, but it is paradoxical that this has all been achieved without an exact understanding of the fundamental pathogenesis and pathophysiology of the disease.

It is therefore entirely justified to break fresh scientific ground in chasing the core abnormalities of asthma. We know now that it is a very heterogeneous and complex disease, and one may even question as to whether asthma is a true entity or whether it merely refers to a shared clinical expression of several, only partially overlapping, biological conditions [1]. It is evident from research on the homeostasis and homeokinesis of so-called healthy and diseased states that the latter is becoming more and more likely [2]. There can be little doubt that this is exactly the reason for the plateau in the progress in research on the fundamental mechanisms of asthma in recent decades. It just requires novel approaches for capturing these levels of complexity [2].

Here, we are at a clear crossroads of scientific paradigms or - better - synergistic parallel routes in cracking bio-clinical problems. The basis remains sound and reliable, hypothesis-testing research. We have all been trained to perform this, using Koch's postulates. This has brought us to where we are, which is an amazing achievement. It is based on measuring properly selected parameters based on careful generation and testing of concepts in relation to the causes and treatment of disease, in this case asthma. The novelty at the present time is that the technical capabilities for generating hypotheses have grown enormously. Hence, rather than strictly searching for the postulated causative pathways of a disease from what is known, high-throughput technologies now allow us to include the discovery of the entirely unknown [3]. This not only raises the chance of finding the unexpected, but is also getting to grip with the biological complexity of disease in individual patients. Asthma patients are not all alike, and individual phenotyping (description by empiricism) followed by endotyping (framing by understanding) seems to be the most effective paradigm for achieving personalised medicine [4]. Can this be done in respiratory medicine and for asthma in particular? The answer is clearly "yes". It requires carefully defining the concepts [5] and the

Received: Dec 312016 | Accepted: Jan 032017

Conflict of interest: None declared.

Copyright CERS 2017 
high-throughput "omics" methodologies [6]. Has it been embraced generally yet? The answer is "no", mainly because such approaches have been colourfully labelled as "fishing expeditions".

In the present issue of the European Respiratory Journal, ReInKE et al. [7] are bravely taking the risk. They set out to identify metabolomic signatures in peripheral blood that: 1) differ between patients with asthma and controls and 2) relate to disease severity. This cross-sectional study was performed in 54 patients (including those with mild, moderate and severe asthma) and 22 healthy controls by using liquid chromatography-mass spectrometry validated by MS/MS or targeted assays. The data pre-processing and statistical algorithms were carefully chosen in an attempt to suppress the big phantom of all "omics": false discovery.

What did they observe? Among the 66 reproducibly detected metabolites, eight appeared to be significantly increased and six significantly decreased in mild asthma as compared to controls, while 22 metabolites significantly contributed to the separation of severe asthma from all other subgroups. Interestingly, the metabolites included exogenous ones, such as dietary lipids or those associated with the gut microbiome, which were mostly involved in separating mild asthma from controls. However, other metabolites, such as S-1-P(d18:1), oleoylethanolamide and $N$-palmitoyltaurine, appeared to be associated with asthma severity. Not unexpectedly, the steroid metabolites cortisone, cortisol and DHEA-S were decreased in asthmatics. This was most pronounced in the five asthmatics using systemic steroids, but there was no significant interaction between inhaled steroid dose and metabolite levels. In addition, steroid-naïve mild asthmatics also exhibited a different metabolic profile to controls. The data suggest that the metabolic signatures of mild asthma differ from those of moderate and severe asthma, the latter being associated with steroid usage and activity of G-protein coupled receptors (GPCR) and/or vagal nerve transient receptor potential vanilloid type 1 receptors (TRPV1).

What is the value of these findings? First, this was an unbiased or - better - unsupervised study design. This means that there was no a priori selection of likely candidate metabolites based on previous knowledge, which makes this a true discovery study. This is greatly needed, because we are more and more stuck with regard to unravelling the determining molecular pathways in asthma. Second, this is metabolomics. Why choose this, as opposed to other "omics" platforms, such as transcriptomics or proteomics? Metabolites represent the downstream signals of everything that is occurring biologically, from gene transcription and gene translation in their complex interaction with the environment [8]. This is actually capturing "real-life" health and disease in all its day-to-day interplay. Of course, it is merely a snapshot, and does not include temporal variation [2], but still, it is the end result of the downstream metabolic processes, and that is what actually counts for a living organism here and now.

The study did not include an independent validation cohort. This can be regarded as a significant limitation. However, let's face it: it may not be surprising that the few other metabolomic studies in asthma are not coming up with identical results [9]. The individual variability in host characteristics and environmental exposures of patients with asthma is likely to be huge, so we had better get used to findings that cannot be fully reproduced between and even within populations. Does this invalidate these studies? I think not. These data represent the first steps in getting some grip on the biological complexities of asthma, which are no longer hidden now we are able to measure them [2,5]. Indeed, the inter- and perhaps even intra-subject variability is much larger than we would have liked, but let us be pleased: there is a lot of new signal amongst the inevitable noise! Validation in an independent sample will strengthen our understanding of the shared signals between populations, but any future failure of validation may also be driven by meaningful (!) inter-individual variability. Let us get used to individual phenotyping in addition to searching the shared denominators of disease.

These novel studies require a level of scrutiny that is unprecedented. ReINKE et al. [7] took several measures to stringently limit the likelihood of false discovery by chance. They only included metabolites that were reproducibly confirmed by MS/MS. However, they also had to make several choices that will have influenced the results, such as abolishing outliers beyond three standard deviations away from the rest of the samples. In addition, metabolites with $>20 \%$ missing values or a relative standard deviation of the quality control samples of $>25 \%$ were disregarded. Finally, the sample size was more or less arbitrary (because of the very few precedent studies), and the significance levels in the univariate and (composite) multivariable analyses were set at 0.05 . These are all choices that affect the outcome. Is this a reason for abandoning these kinds of studies? No, it is not! Even in the absence of a validation cohort, false discovery can adequately and transparently be estimated and dealt with by strictly using detailed and stepwise recommendations regarding 1) quality control of the assays [10], 2) experimental design and analysis [11] and 3) multivariable modelling [12].

Where are we now? Together with the new "omics" platforms, study designs and statistical algorithms have now reached an acceptable standard for a real step change in biomedical science. The data are 
coming in and the present study by ReINKE et al. [7] in asthma is a very good example. The findings are opening our eyes, with a glance at the as yet undiscovered pathways that appear to be linked to asthma. These kinds of study have the best chance of traversing the plateau in medical progress by adding "omics" and machine learning to our clinical repertoire [13], perhaps even at the point of care [14]. This may, in particular, benefit research and the clinical management of chronic, non-communicable diseases [15]. We are starting to capture the complexity (perhaps even beauty) of human beings under various conditions when adapted (health) or not (disease) to our environment. This is the result of a solid and modern fishing expedition. And the good asthma catch tastes for more!

\section{References}

$1 \quad$ Sterk PJ. Chronic diseases like asthma and COPD: do they truly exist? Eur Respir J 2016; 47: 359-361.

2 Thamrin C, Frey U, Kaminsky DS, et al. Systems biology and clinical practice in respiratory medicine: the twain shall meet. Am I Respir Crit Care Med 2016; 194: 1053-1061.

3 Chen R, Mias GI, Li-Pook-Than J, et al. Personal omics profiling reveals dynamic molecular and medical phenotypes. Cell 2012; 148: 1293-1307.

4 Anderson GP. Endotyping asthma: new insights into key pathogenetic mechanisms in a complex heterogenous disease. Lancet 2008; 372: 1107-1119.

5 Agusti A, Anto JM, Auffray C, et al. Personalized respiratory medicine: exploring the horizon, addressing the issues. Summary of a BRN-AJRCCM workshop held in Barcelona on June 12, 2014. Am J Respir Crit Care Med 2015; 191: 391-401.

6 Wheelock CE, Goss VM, Balgoma D, et al. Application of 'omics technologies to biomarker discovery in inflammatory lung diseases. Eur Respir J 2013; 42: 802-825.

7 Reinke SN, Gallart-Ayala H, Gomez C, et al. Metabolomic analysis identifies different metabotypes of asthma severity. Eur Respir J 2017; 49: 1601740

8 Kelly RS, Dahlin A, McGearchie MJ, et al. Asthma metabolomics and the potential for integrative omics in research and the clinic. Chest 2017; 151: 262-277.

9 Checkley W, Deza MP, Klawitter J, et al. Identifying biomarkers for asthma diagnosis using targeted metabolomics approaches. Respir Med 2016; 121: 59-66.

10 Dunn WB, Wilson ID, Nicholls AW, et al. The importance of experimental design and QC samples in large-scale and MS-driven untargeted metabolomic studies of humans. Bioanalysis 2012; 18: 2249-2264.

11 McShane LM, Cavenagh MM, Lively TG, et al. Criteria for the use of omics-based predictors in clinical trials: explanation and elaboration. BMC Med 2013; 11: 220.

12 Collins GS, Reitsma JB, Altman DG, et al. Transparent reporting of a multivariable prediction model for individual prognosis or diagnosis (TRIPOD): the TRIPOD statement. BMJ 2015; 350: 350:g7594.

13 Obermeyer Z, Emanuel EJ. Predicting the future - big data, machine learning and clinical medicine. $N$ Engl J Med 2016; 375: 1216-1219.

14 Bos LD, Sterk PJ, Fowler SJ. Breathomics in the setting of asthma and chronic obstructive pulmonary disease. J Allergy Clin Immunol 2016; 138: 970-976.

15 Cesario A, Auffray C, Agusti A, et al. A systems medicine clinical platform for understanding and managing non-communicable diseases. Curr Pharm Des 2014; 20: 5945-5956. 